\title{
Emprego dos marcadores de prognóstico no tratamento para o carcinoma invasor de colo
}

\author{
Use of the prognosis markers in the treatment for the invasive \\ cervical carcinoma
}

Revisão

Palavras-chave

\section{Resumo}

Neoplasias do colo do útero/diagnóstico Neoplasias do colo do útero/terapia

Prognóstico

Estadiamento de neoplasias

Metástase linfática Invasividade neoplásica

Keywords

Uterine cervical neoplasms/diagnosis Uterine cervical neoplasms/therapy

Prognosis

Neoplasm staging

Lymphatic metastasis

Neoplasm invasiveness

carcinoma invasor do colo uterino representa um grande problema de Saúde Pública, especialmente nos países em desenvolvimento. $\bigcirc$ seu tratamento, baseado na histerectomia radical, radioterapia e/ou quimioterapia, apresenta uma morbidade considerável. Os marcadores prognósticos devem ser considerados no planejamento terapêutico, de forma a otimizar os resultados, diminuir as complicações e aumentar a sobrevida das pacientes. São considerados marcadores prognósticos o estadiamento, o tamanho tumoral, o tipo histológico, o grau de diferenciação, a invasão linfovascular, a profundidade da invasão estromal, a presença de metástases linfonodais e o acometimento de margens cirúrgicas. Esse estudo visou fazer uma revisão da literatura em relação à utilização desses marcadores no planejamento terapêutico das mulheres com carcinoma invasor do colo uterino. $\bigcirc$ tratamento baseado nesses marcadores pode apresentar melhores resultados, com menor taxa de complicações e melhora na sobrevida das pacientes.

\section{Abstract}

The uterine cervix invasive carcinoma represents a major public health problem, mainly in the developing countries. Its treatment, based on radical hysterectomy, radiotherapy and/or chemotherapy presents a considerable morbidity. Prognostic markers should be taken into consideration in the therapeutic planning, so that the results would be optimized, complications reduced, and patients' survival prolonged. Accepted prognostic markers are: stage, tumoral size, histological type, degree of differentiation, lymphovascular invasion, depth of the stromal invasion, presence of lymph nodal metastases, and surgical margins involvement. This study aims at making a literature review concerning the use of theses markers in the therapeutic planning of women with uterine cervix invasive carcinoma. The treatment based on these markers may present better results, with lower ratio of complications and an improvement in the patients' survival.

Correspondência:

Agnaldo Lopes Silva Filho

Departamento de Ginecologia e Obstetrícia da Faculdade de Medicina da Universidade Federal de Minas Gerais Avenida Professor Affredo Balena, 190 - Santa Efigênia (EP 30130-100 - Belo Horizonte (MG), Brasi Fone: (31) 3409-9764/Fax: (55-31) 3409-9765 E-mail: agsilvaf@terra.com.br

Recebido $30 / 7 / 09$

Aceito com modificacões
' Professor Adjunto do Departamento de Ginecologia e Obstetrícia e Anatomia Patológica da Universidade Federal de Minas Gerais - UFMG - Belo Horizonte (MG), Brasil. 


\section{Introdução}

O carcinoma invasor do colo uterino representa um grande problema de Saúde Pública, com cerca de 490.000 novos casos e 270.000 mortes ao ano no mundo ${ }^{1}$. A maioria dos casos ocorre nos países em desenvolvimento, devido à precariedade dos programas de rastreamento ${ }^{1}$. No Brasil, estimou-se a ocorrência de 18.680 casos em 2008, com um risco estimado de 19 casos a cada 100.000 mulheres $^{2}$. Representa a terceira neoplasia maligna mais comum entre as mulheres e a quarta causa de morte nesse grupo ${ }^{2}$.

O estadiamento tumoral, conforme preconizado pela Federação Internacional de Ginecologia e Obstetrícia (FIGO), representa o principal determinante para a escolha do tratamento no carcinoma invasor de colo uterino ${ }^{3}$. A sobrevida em cinco anos de pacientes com câncer de colo uterino estádio IB varia de 65 a 95\%, o que motivou a pesquisa de fatores prognósticos que justificassem essa variação ${ }^{4}$. Constituem fatores prognósticos no carcinoma invasor do colo uterino o tamanho tumoral, o tipo histológico, o grau de diferenciação, a invasão linfovascular (ILV), a profundidade da invasão estromal, a presença de metástases linfonodais e o acometimento de margens cirúrgicas ${ }^{5}$.

\section{Estadiamento}

O estadiamento representa o fator prognóstico mais importante no carcinoma invasor do colo uterino, refletindo sua disseminação local e à distância ${ }^{6}$. A taxa de sobrevida em cinco anos varia entre $97,5 \%$, nos tumores estádio IA1, e 9,3\%, no estádio IVB ${ }^{6}$. Constitui o maior determinante para o planejamento terapêutico em mulheres com carcinoma invasor do colo uterino (Figuras 1 e 2). No entanto, a presença de outros fatores de risco deve ser considerada, principalmente no emprego de terapia adjuvante ${ }^{3}$.

No estádio IA1, a ocorrência de acometimento linfonodal é de cerca de $1 \%$, tornando desnecessária a linfadenectomia ${ }^{7}$. A histerectomia extrafascial constitui o tratamento padrão para mulheres com prole definida ${ }^{3}$. Para mulheres que optam pela preservação da fertilidade, a conização com bisturi ou laser pode representar uma opção terapêutica ${ }^{\top}$ (Figura 1).

No estádio IA2, a presença ou ausência de ILV deve ser considerada para definição terapêutica ${ }^{3}$. A traquelectomia radical com linfadenectomia pélvica pode ser levada em conta para pacientes que desejam a preservação da fertilidade $^{3}$. Para aquelas sem desejo de manutenção do futuro reprodutivo, o tratamento cirúrgico inclui desde a histerectomia extrafascial até a histerectomia radical e a linfadenectomia (LN) pélvica ${ }^{3}$ (Figura 1 ). A radioterapia é reservada para pacientes que não apresentam condições cirúrgicas ${ }^{8}$. Nesses casos, a adição de um agente quimiossensibilizante (mais comumente, a cisplatina) melhora em $10 \%$ a sobrevida em cinco anos nestas pacientes?.

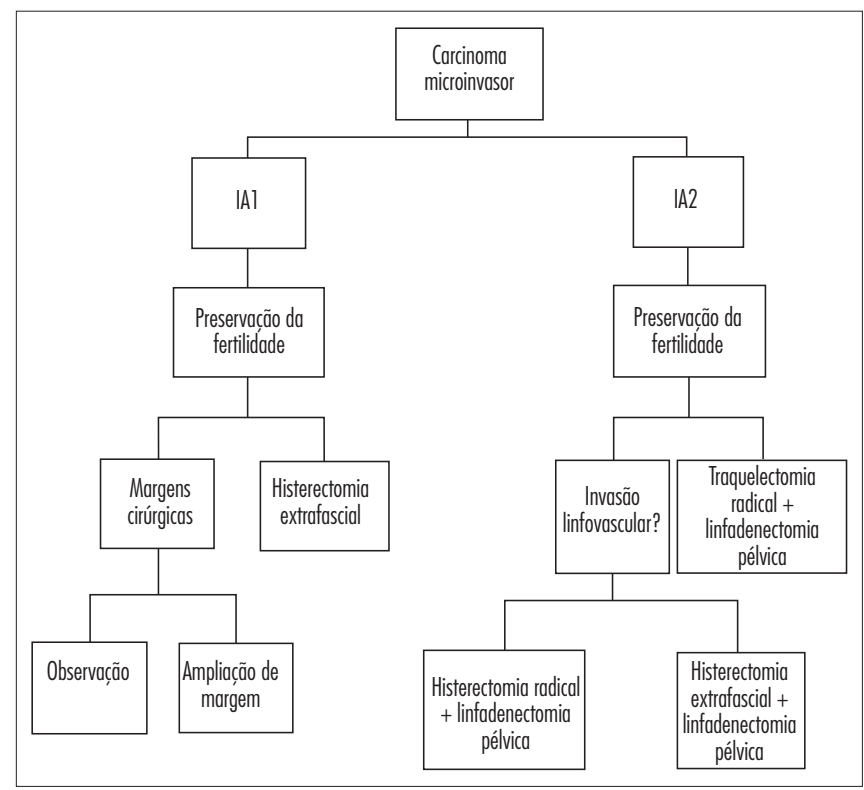

Figura 1 - Abordagem das pacientes com neoplasias microinvasoras do colo uterino.

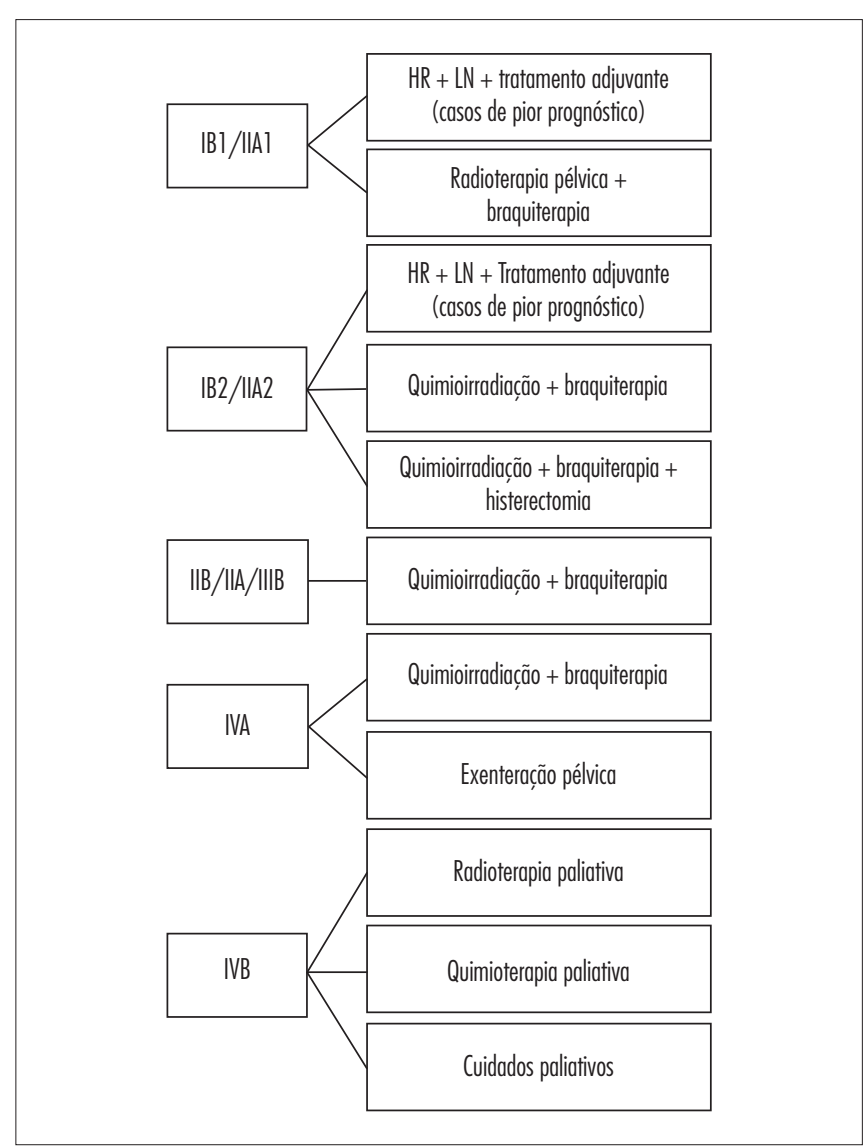

Figura 2 - Abordagem das pacientes com carcinoma invasor do colo uterino. 


\section{Estádios IB e IIA}

Nos estádios IB1 e IIA, a maior parte dos estudos indica que o tratamento radioterápico (radioterapia externa e braquiterapia) e o tratamento cirúrgico (histerectomia radical e linfadenectomia pélvica) têm resultados semelhantes quanto à sobrevida ${ }^{6}$. A escolha entre essas formas de tratamento deve se basear na idade, nas condições clínicas da paciente e na presença de fatores prognósticos, porque a combinação de tratamentos aumenta muito a morbidade terapêutica ${ }^{10}$ (Figura 2). $\mathrm{O}$ tratamento cirúrgico apresenta como vantagem a identificação e o tratamento de doença extrauterina não suspeitada e o exame histopatológico da peça cirúrgica, importantes no estabelecimento do prognóstico e na indicação de terapia adjuvante, além da possibilidade de preservação da função ovariana e sexual ${ }^{10}$. A radioterapia tem como principal benefício a fácil administração em pacientes com risco cirúrgico aumentado ${ }^{10}$.

Há um acréscimo de $10 \%$ na sobrevida em cinco anos com a quimioirradiação como tratamento exclusivo em relação ao tratamento radioterápico isolado?.

\section{Estádios IIB a IV}

No caso de tumores estádios IIB e IVA é indicada a radioterapia externa e a braquiterapia ${ }^{3}$ (Figura 2). Quando comparada à radioterapia isolada, a quimioirradiação está associada ao aumento na sobrevida em cinco anos de $7 \%$ no estádio IIB e de $3 \%$ nos estádios III a IVA . A exenteração pélvica pode ser uma opção para pacientes com tumores no estádio IVA sem envolvimento parametrial e vaginal que apresentem condições clínicas para a intervenção ${ }^{3}$. Pacientes com doença metastática (IVB) devem receber tratamento sintomático ou quimioterapia e/ou radioterapia como proposta paliativa ${ }^{3}$ (Figura 2).

\section{Tamanho tumoral}

O diâmetro tumoral tem sido considerado um fator prognóstico relevante para mulheres nos estádios $\mathrm{I}$ e $\mathrm{II}^{10}$. A sobrevida é de $95 \%$ para tumores menores que $2 \mathrm{~cm}$; $79 \%$ para tumores entre 2 e $3 \mathrm{~cm}$; e $47 \%$ quando maiores que $4 \mathrm{~cm}^{11}$. A forma do crescimento tumoral é determinante da sobrevida, sendo que os tumores barrel-shape são associados a pior prognóstico ${ }^{12}$.

As opções terapêuticas das mulheres com tumores com diâmetro superior a $4 \mathrm{~cm}$ incluem histerectomia radical associada à LN pélvica, quimioirradiação ou quimioirradiação associada à histerectomia adjuvante ${ }^{8}$ (Figura 2). As pacientes submetidas à cirurgia como tratamento primário se beneficiam de terapia adjuvante na presença de ILV ou invasão estromal maior que um terço ${ }^{13}$ Estudos sugerem que a quimiorradiação seja superior à radioterapia nesse grupo de pacientes?

\section{Tipo histológico}

Os tumores não epidermoides apresentam pior prognóstico em comparação ao carcinoma de células escamosas $(\mathrm{CCE})^{5,6}$. A taxa de sobrevida em cinco anos de pacientes nos estádios IB e IIA tratadas cirurgicamente é de 72,2\% para os tumores adenoescamosos ou adenocarcinoma, e de $81,2 \%$ para os tumores escamosos ${ }^{14}$.

Acredita-se que essa diferença possa ser resultado de maior resistência à radioterapia, propensão ao acometimento intraperitoneal e maior incidência de metástase linfonodal e à distância ${ }^{5}$. A ocorrência de metástase ovariana é maior nos adenocarcinomas e está associada ao tamanho do tumor, enquanto nos tumores escamosos, a metástase ovariana se associa ao estádio da doença ${ }^{5}$.

Pacientes jovens com CCE são candidatas à preservação dos ovários ${ }^{3}$. Nos adenocarcinomas a ooforectomia deve ser realizada, pelo risco de até $5,3 \%$ para metástase ovariana ${ }^{15}$.

O tipo histológico não define o planejamento terapêutico isoladamente ${ }^{3}$. No entanto, naquelas mulheres submetidas à radioterapia adjuvante pela presença de outros fatores de risco, a redução das taxas de recorrência local foi mais acentuada nos casos de tumores adenoescamosos e nos adenocarcinomas em comparação ao $\mathrm{CCE}^{13}$.

\section{Grau de diferenciação}

O grau de diferenciação como fator prognóstico independente é controverso, principalmente pela falta de padronização e alta subjetividade evidenciadas fracas pela concordância intra e interobservador ${ }^{5,16}$. Enquanto alguns autores correlacionam o grau de diferenciação histológica com o pior prognóstico, outros não encontram diferença na sobrevida livre de doença após ajuste com outros fatores, como metástase linfonodal e tamanho tumoral ${ }^{17}$. Sua classificação deve ser estimulada; entretanto, não deve alterar o tratamento ${ }^{3}$.

\section{Invasão linfovascular}

O diagnóstico da presença de ILV é menos sujeito a falhas, apresentando uma concordância intra e interobservador muito boa ${ }^{16}$. A presença de ILV está associada à metástase nos linfonodos pélvicos, ao acometimento parametrial e vaginal ${ }^{18}$.

No estádio IA2, a presença de ILV indica a realização de histerectomia radical e linfadenectomia pélvica. Aquelas sem ILV são candidatas à histerectomia extra-fascial ${ }^{3}$ (Figura 1). Nos carcinomas invasores do colo uterino, a presença de ILV associada ao diâmetro tumoral maior que $4 \mathrm{~cm}$ e/ou 
invasão estromal maior que um terço indica radioterapia adjuvante com melhora nas taxas de recorrência, tempo livre de doença e sobrevida ${ }^{13}$.

\section{Profundidade da invasão estromal}

A profundidade da invasão estromal pode ser definida em termos absolutos por milímetros ou por terços de invasão uterina ${ }^{5}$. Esse achado histopatológico constitui um fator prognóstico independente, associado à sobrevida livre de doença e à presença de metástase linfonodal 5 .

Em pacientes nos estádios IB e IIA submetidas a tratamento cirúrgico, a presença de invasão estromal maior que um terço associada à ILV e/ou tumor maior que $4 \mathrm{~cm}$ indica a radioterapia adjuvante ${ }^{13}$

\section{Metástase linfonodal}

O acometimento linfonodal é um dos fatores histopatológicos mais importantes no prognóstico do carcinoma invasor do colo uterino ${ }^{5}$. Pacientes nos estádios IB e IIA apresentam sobrevida em cinco anos de $95 \%$ na ausência de metástase linfonodal e de $78 \%$ na presença deste fator ${ }^{19}$. O número de linfonodos e os segmentos acometidos também são de grande importância ${ }^{5}$. A taxa de sobrevida em cinco anos é de $85 \%$ e $50 \%$ para as pacientes que apresentaram apenas um e dois ou mais linfonodos comprometidos, respectivamente ${ }^{19}$. O acometimento de linfonodos para-aórticos e ilíacos foi associado à sobrevida de $20 \%$, enquanto o comprometimento de linfonodos abaixo desse nível foi associado à sobrevida de $84 \%$ em cinco anos ${ }^{19}$.

A histerectomia radical nos casos com acometimento linfonodal diagnosticado durante a intervenção cirúrgica é controversa ${ }^{20}$. No entanto, a associação entre a histerectomia radical e a radioterapia adjuvante aumenta a morbidade e não modifica a sobrevida em relação aos casos em que o procedimento é abortado ${ }^{3,20}$.

Nos casos de diagnóstico histopatológico de metástase linfonodal pélvica, a quimioirradiação adjuvante está associada à diminuição nas taxas de recidiva local e ao aumento na sobrevida em cinco anos ${ }^{21}$. Caso haja acometimento de linfonodos para-aórticos, é indicada a radioterapia de campo estendido associada à cisplatina ${ }^{8}$ (Figura 3).

\section{Margens cirúrgicas}

Mulheres submetidas à histerectomia radical com acometimento parametrial e/ou margens vaginais comprometidas ou inferiores a $0,5 \mathrm{~cm}$ apresentam maior taxa de recorrência tumoral e menor tempo livre de doença e sobrevida em cinco anos ${ }^{22,23}$.
Pacientes com diagnóstico de carcinoma microinvasor que apresentam as margens do cone comprometidas por lesão microinvasora ou intraepitelial de alto grau apresentam risco aumentado para neoplasia residual ${ }^{24}$. Nesses casos, é indicada a realização de nova conização para definição da gravidade da lesão e planejamento terapêutico ${ }^{24}$ (Figura 1).

Para as pacientes com carcinoma invasor do colo uterino submetidas à histerectomia radical com diagnóstico de acometimento parametrial e/ou margens cirúrgicas comprometidas ou exíguas (menor que $0,5 \mathrm{~cm}$ ), é indicada a quimioirradiação ${ }^{21,25}$.

\section{Marcadores moleculares}

As células neoplásicas demonstram algumas características biológicas, como evasão da apoptose, resistência aos fatores inibidores de crescimento, emissão de seus próprios sinais mitogênicos, perda da diferenciação, aquisição de neovascularização e, em casos de tumores

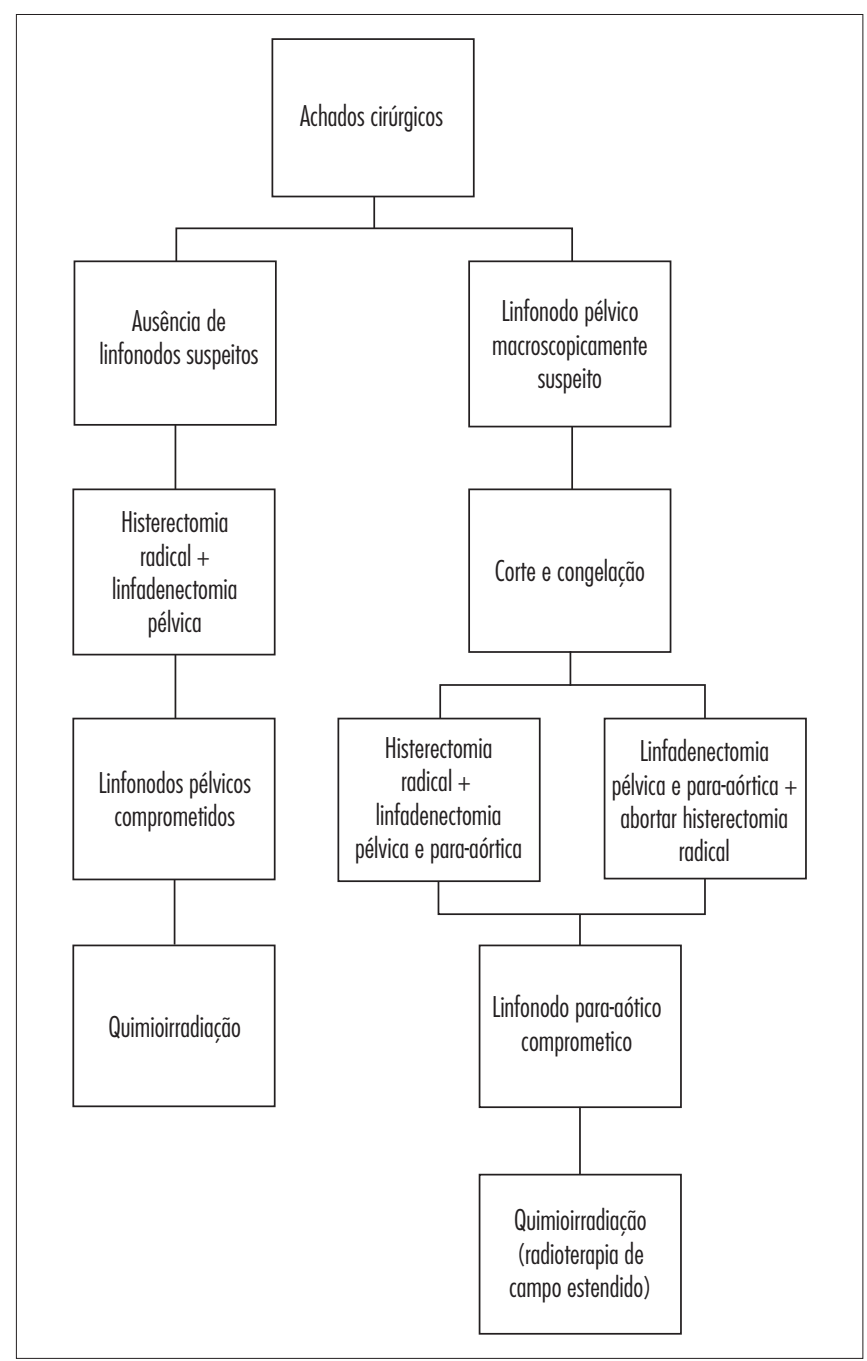

Figura 3 - Abordagem de pacientes com carcinoma invasor do colo uterino baseada no acometimento linfonodal. 
mais avançados, capacidade de invasão e metastatização ${ }^{26}$. A identificação de novos marcadores tumorais pode ser valiosa no seguimento das pacientes, para a definição daquelas que se beneficiariam de terapia adjuvante ou de um tratamento primário mais agressivo ${ }^{26}$.

O estudo de marcadores moleculares no carcinoma invasor do colo uterino é promissor, mas, até o momento, não apresenta aplicação na prática clínica. A expressão das proteínas p53, KI-67 e CD31 e da atividade da telomerase no tumor e nas margens vaginais de pacientes submetidas a tratamento cirúrgico para neoplasia invasora do colo uterino parecem se associar a outros fatores prognósticos ${ }^{27-29}$. O papel das micrometástases parametriais, identificadas por imunohistoquímica para citoqueratinas, em pacientes com paramétrios livres ao exame histopatológico, necessita de melhor definiçãa ${ }^{30}$.

\section{Referências}

1. International Agency for Research on Cancer - IARC [Internet]. Cancer incidence, mortality and prevalence worldwide. Lyon: IARC; 2009 [cited 2009 Jul 19]. Available form: http://wwwdep.iarc.fr/

2. Ministério da Saúde. Instituto Nacional do Câncer [Internet]. Estimativa de incidência e mortalidade por câncer no Brasil. Brasília (DF): INCA; c2008 [citado 2009 Jul 17]. Disponível em: http://www.inca.gov.br/estimativa.

3. Denny L, Hacker NF, Gori J, Jones III HW, Ngan HYS, Pecorelli S. Staging classifications and clinical practice guidelines for gynaecologic cancers [Internet]. London: International Federation of Gynecology and Obstetrics. Committee on Gynecologic Oncology; 2006 [cited 2009 Jul 19]. Available from: http://www.figo.org/ publications

4. Creasman WT, Kohler MF. Is lymph vascular space involvement an independent prognostic factor in early cervical cancer? Gynecol Oncol. 2004 Feb;92(2):525-9.

5. Singh N, Arif S. Histopathologic parameters of prognosis in cervical cancer-a review. Int J Gynecol Cancer. 2004; 14(5):741-50.

6. Quinn MA, Benedet JL, Odicino F, Maisonneuve P, Beller U, Creasman WT, et al. Carcinoma of the cervix uteri. FIGO 6th Annual Report on the Results of Treatment in Gynecological Cancer. Int J Gynaecol Obstet. 2006;95 Suppl 1:S43-103.

7. Raspagliesi F, Ditto A, Quattrone P, Solima E, Fontanelli R, Dousias $V$, et al. Prognostic factors in microinvasive cervical squamous cell cancer: long-term results. Int J Gynecol Cancer. 2005;15(1):8893.

8. National Comprehensive Cancer Network [Internet]. Clinical practice guidelines in oncology. Cervical cancer. Washington: NCCN; 2009 [cited 2009 Jul 12] ]. Available from: http://www. nccn.org/professionals/physician_gls/f_guidelines.asp

9. Vale C, Tierney JF, Stewart LA, Brady M, Dinshaw K, Jakobsen A, et al. Chemoradiotherapy for Cervical Cancer Meta-Analysis Collaboration. Reducing uncertainties about the effects of chemoradiotherapy for cervical cancer: a systematic review and meta-analysis of individual patient data from 18 randomized trials. J Clin Oncol. 2008;26(35):5802-12.

10. Landoni F, Maneo A, Colombo A, Placa F, Milani R, Perego P, et al. Randomised study of radical surgery versus radiotherapy for stage Ib-lla cervical cancer. Lancet. 1997;350(9077): 535-40.

11. Robert ME, Fu YS. Squamous cell carcinoma of the uterine cervix: a review with emphasis on prognostic factors and unusual variants. Semin Diagn Pathol. 1990;7(3):173-81.

12 Trimbos JB, Lambeek AF, Peters AA, Wolterbeek R, Gaarenstroom $K N$, Fleuren GJ, et al. Prognostic difference of surgical treatment of exophytic versus barrel-shaped bulky cervical cancer. Gynecol Oncol. 2004;95(1):77-81.

13. Rotman M, Sedlis A, Piedmonte MR, Bundy B, Lentz SS, Muderspach $\mathrm{LI}$, et al. A phase III randomized trial of postoperative pelvic irradiation in Stage IB cervical carcinoma with poor prognostic features: follow-up of a gynecologic oncology group study. Int J Radiat Oncol Biol Phys. 2006;65(1):169-76.

14. Lai $\mathrm{CH}$, Hsueh S, Hong JH, Chang TC, Tseng CJ, Chou HH, et al. Are adenocarcinomas and adenosquamous carcinomas different from squamous carcinomas in stage IB and II cervical cancer patients undergoing primary radical surgery? Int J Gynecol Cancer. $1999 ; 9(1): 28-36$.

15. Shimada M, Kigawa J, Nishimura R, Yamaguchi S, Kuzuya K, Nakanishi T, et al. Ovarian metastasis in carcinoma of the uterine cervix. Gynecol Oncol. 2006;101(2):234-7.

16. Noviello MB, Silva-Filho AL, Traiman P, Triginelli SA, Noviello $M$, Pedrosa MS, et al. Inter- and intraobserver variability in the assessment of tumor grade and lymphovascular space invasion in patients with squamous cell carcinoma of the cervix. Eur J Obstet Gynecol Reprod Biol. 2008;138(2):246-8.

17. Stock RJ, Zaino R, Bundy BN, Askin FB, Woodward J, Fetter B, et al. Evaluation and comparison of histopathologic grading systems of epithelial carcinoma of the uterine cervix: Gynecologic Oncology Group studies. Int J Gynecol Pathol. 1994;13(2): 99-108.

18. Silva-Filho AL, Reis FM, Traiman P, Pedrosa MS, Miranda D, Triginelli SA. Clinicopathological features influencing pelvic lymph node metastasis and vaginal and parametrial involvement in patients with carcinoma of the cervix. Gynecol Obstet Invest. 2005;59(2):92-6.

19. Kim SM, Choi HS, Byun JS. Overall 5-year survival rate and prognostic factors in patients with stage IB and IIA cervical cancer treated by radical hysterectomy and pelvic lymph node dissection. Int J Gynecol Cancer. 2000;10(4):305-312.

20. Richard SD, Krivak TC, Castleberry A, Beriwal S, Kelley JL 3rd, Edwards RP, et al. Survival for stage IB cervical cancer with positive lymph node involvement: a comparison of completed vs. abandoned radical hysterectomy. Gynecol Oncol. 2008;109(1):43-8.

21. Peters WA 3rd, Liu PY, Barrett RJ 2nd, Stock RJ, Monk BJ, Berek JS, et al. Concurrent chemotherapy and pelvic radiation therapy compared with pelvic radiation therapy alone as adjuvant therapy after radical surgery in high-risk early-stage cancer of the cervix. J Clin Oncol. 2000;18(8):1606-13.

22. Estape ER, Angioli R, Madrigal M, Janicek M, Gomez C, Penalver $M$, et al. Close vaginal margins as a prognostic factor after radical hysterectomy. Gynecol Oncol. 1998;68(3):229-32. 
23. Wright JD, Grigsby PW, Brooks R, Powell MA, Gibb RK, Gao F, et al. Utility of parametrectomy for early stage cervical cancer treated with radical hysterectomy. Cancer. 2007;1 10(6):1281-6.

24. Phongnarisorn C, Srisomboon J, Khunamornpong S, Siriaungkul S, Suprasert $\mathrm{P}$, Charoenkwan K, et al. The risk of residual neoplasia in women with microinvasive squamous cervical carcinoma and positive cone margins. Int J Gynecol Cancer. 2006; 16(2):655-9.

25. Viswanathan AN, Lee $H$, Hanson $E$, Berkowitz RS, Crum CP. Influence of margin status and radiation on recurrence after radical hysterectomy in Stage IB cervical cancer. Int J Radiat Oncol Biol Phys. 2006;65(5):1501-7.

26. Hahn WC, Weinberg RA. Rules for making human tumor cells. N Engl J Med. 2002;347(20):1593-603.

27. Silva-Filho AL, Traiman $P$, Triginelli SA, Reis FM, Pedrosa MS, Miranda D, et al. Expression of p53, Ki-67, and CD31 in the vaginal margins of radical hysterectomy in patients with stage IB carcinoma of the cervix. Gynecol Oncol. 2004;95(3):646-54.

28. Santos-Filho AS, Triginelli SA, Traiman P, Cunha-Melo JR, SilvaFilho AL. Missing association between telomerase activity and clinicopathological features in patients with early stage carcinoma of the cervix. Arch Gynecol Obstet. 2007;275(1):13-7.

29. Silva-Filho AL, Traiman P, Triginelli SA, Reis FM, Pedrosa MS, Miranda D, et al. Association between CD3 1 expression and histopathologic features in stage IB squamous cell carcinoma of the cervix. Int J Gynecol Cancer. 2006; 16(2):757-62.

30. Cândido EB, Silva-Filho AL, Triginelli SA, Pedrosa MS, Macarenco $R$, Porto $L$, et al. Histopathological and immunohistochemical (cytokeratins AE 1/AE3) study of the parametrium of patients with early stage cervical cancer. Eur J Obstet Gynecol Reprod Biol. $2008 ; 141(1): 58-63$ 